\title{
Clinical features and outcomes of pregnant women with COVID-19: a systematic review and meta-analysis
}

\author{
Yijie Gao ${ }^{1}$, Lei Ye ${ }^{1}$, Jia-shuo Zhang ${ }^{1}$, Yang-xue Yin², Min Liu $^{2}$, Hong-biao Yu ${ }^{2}$, and Rong \\ Zhou $^{2}$ \\ ${ }^{1}$ Affiliation not available \\ ${ }^{2}$ Sichuan University West China Second University Hospital
}

April 28, 2020

\begin{abstract}
Background: COVID-19 outbreak in Wuhan, China recently. It quickly spread throughout. Objectives: To investigate clinical features and outcomes of pregnant women with COVID-19. Search Strategy: PubMed - Web of Science、EMBASE、MEDLINE were searched from January 1, 2020 to April 16, 2020. Selection Criteria: Case review of pregnant women infected with COVID19 Data Collection and Analysis: Two reviewers screened 366 literatures and 14 studies included, four reviewers independently extracted the features of the literatures. We used random-effects model to analyze incidence (P) and $95 \%$ confidence interval (95\%CI). Heterogeneity was assessed using the I2 statistic. Main Results: The meta- analysis included 236 pregnant women with COVID-19. The results were as follows: positive CT findings (71\%; 95\%CI, 0.49 0.93), cesarean section (65\%; 95\%CI, $0.42^{\sim} 0.87$ ), fever (51\%; 95\%CI, 0.35 0.67), lymphopenia (49\%; 95\%CI, 0.29 0.70), coexisting disorders (33\%; 95\%CI, 0.21 0.44), cough $(31 \%$; 95\% CI, 0.23 0.39), fetal distress (29\%; 95\%CI, 0.08 0.49), preterm labor (23\%; 95\%CI, 0.14 0.32), severe case or death $(12 \% ; 95 \% \mathrm{CI}, 0.03 \sim 0.20)$. The subgroup analysis showed compared with nonpregnant patients, pregnant women with COVID-19 had significantly lower incidences of fever (pregnant women group, 51\%; nonpregnant patients group, 91\%; $\mathrm{P}_{\mathfrak{j}} 0.00001$ ) and cough (pregnant women group, 31\%; nonpregnant patients group, $67 \% ; \mathrm{P} j 0.0001$ ). Conclusions: The incidence of fever, cough and positive CT findings in pregnant women with COVID-19 is less than that in the general population, preterm labor is the opposite. There is no evidence that COVID-19 can propagate vertically for the time being. Keywords: 2019-nCoV, COVID-19, clinical features, pregnancy outcomes, pregnant women, meta-analysis
\end{abstract}

\section{Introduction}

The unexplained clustering pneumonia cases related to the south China seafood wholesale market were reported in Wuhan City, Hubei Province, China in December 2019(1). China scientists isolated novel coronavirus from patients and sequenced the genome, and found that the genetic sequence of the virus was at least $70 \%$ similar to that of the human severe acute respiratory syndrome coronavirus (SARS-CoV). World Health Organization (WHO) named this novel coronavirus 2019-nCoV ${ }^{(2)}$ and the 2019-nCoV causes Coronavirus Disease 2019 (COVID-19) $^{(3)}$. As of April 16, 2020, more than 83000 cases have been confirmed in China and 2022000 cases in the world ${ }^{(4)}$.

Both 2019-nCoV and SARS-CoV are $\beta$-coronaviruses. The mortality rate of SARS-CoV infection is $10 \%$, among which the mortality rate of maternal infection is $25 \%{ }^{(5)}$. The clinical outcome of pregnant women is worse than that of non-pregnant women. To date, clinical data on pregnant women infected with $2019-\mathrm{nCoV}$ is still very limited. Therefore, we conducted this systematic review and meta-analysis to assess the clinical features and pregnancy outcomes of pregnant women infected with COVID-19, with a view to help formulate clinical treatment strategies for pregnant women with COVID-19.

\section{Methods}




\subsection{Search strategy}

The protocol for the meta-analysis was based on the MOOSE checklist ${ }^{(6)}$ (Meta-analysis Of Observational Studies in Epidemiology) and EQUATOR Reporting Guidelines ${ }^{(7)}$ (Preferred Reporting Items for Systematic Reviews and Meta-Analyses). We only conducted a literature review, thus ethics approval was not required.

We systematically searched the literature in PubMed, Web of Science, EMBASE, MEDLINE databases. The retrieval period is from January 1, 2020 to April 16, 2020. The search keywords are ((COVID-19) OR (2019 novel coronavirus infection) OR (COVID19) OR (coronavirus disease 2019) OR (coronavirus disease-19) OR (2019-nCoV disease) OR (2019 novel coronavirus disease) OR (2019-nCoV infection) OR (SARS-CoV-2) OR (Wuhan coronavirus) OR (Wuhan seafood market pneumonia virus) OR (COVID19 virus) OR (COVID-19 virus) OR (coronavirus disease 2019 virus) OR (SARS-CoV-2) OR (SARS2) OR (2019-nCoV) OR (2019 novel coronavirus)) AND ((Pregnancy) OR (Pregnancies) OR (Gestation) OR (Pregnant Women) OR (Pregnant Woman) OR (Woman, Pregnant) OR (Women, Pregnant)). The literature has no language restrictions. We used Endnote X7 library (Clarivate Analytics, Philadelphia, PA, USA) to remove duplicate citations and manage the references. We hand-searched bibliographies of retrieved papers for additional references.

\subsection{Selection criteria}

\subsubsection{Inclusion criteria}

1) Case reports, case series and observational studies of pregnant women infected with COVID-19;

2) Describe the clinical features and/or outcomes of the patient and the fetus / newborn.

\subsubsection{Exclusion criteria}

1) Republished literature;

2) Article types are authors' replies, editorials, guidelines;

3) The number of cases is less than 5 in case report, case series and observational studies;

4) Literature with incomplete or missing data.

\subsection{Data extraction and analysis}

\subsubsection{Data extraction}

The two reviewers (LY, JS.Z) independently screened the literature based on the search strategy, inclusion and exclusion criteria, and extracted relevant data. When the opinions were inconsistent, they sought the opinions of the third reviewer (YJ.G) or negotiated solutions.

\subsubsection{Quality assessment}

Four reviewers (YJ.G, YX.Y, ML, HB. Y) independently extracted the following features of the literatures listed in study characteristics: first author, published time, study date, the number and age of patients and the number of severe case or death, fever, cough, lymphopenia, positive CT findings, coexisting disorders, preterm labor, cesarean section, fetal distress, neonatal asphyxia or neonatal death or stillbirth, neonatal infection, virus in breast milk. They also evaluated the quality of the literature using the Institute of Health Economics (IHE) case series methodological quality evaluation tool ${ }^{(8)}$, which is evaluated in 8 areas: (1) Research purpose, (2) Research population, (3) Intervention and joint intervention, (4) Outcome measures, (5) Statistical analysis, (6) Results and conclusions, (7) Conflict of interest and funding sources, (8) New entry. There is a total of 20 items above, satisfying $14(70 \%)$ or more is considered acceptable quality.

\subsubsection{Statistical analysis}

All calculations were performed by Review Manager soft-ware (version 5.3, Nordic Cochrane Centre) and guided by Implement Meta-Analysis with Non-Comparative Binary Data in RevMan Software ${ }^{(9)} . I^{2}$ statistic 
was used to assess heterogeneity. $I^{2}$ of less than $25 \%$ was low heterogeneity, of $25 \% \sim 50 \%$ was medium heterogeneity, and more than $50 \%$ was high heterogeneity. Because of the high heterogeneity of this study, we used random effects model to pool the study-specific frequencies and 95\% confidence interval (95\% CI) of clinical features or outcomes. Cochrane $\mathrm{Q}$ test $P<0.05$ was considered statistically significant. Funnel plots were used to assess publication bias. Subgroup analysis was used to assess sensitivity.

\section{Results}

\subsection{Study selection}

A total of 364 relevant documents were retrieved by the search methods above, including 103 articles from PubMed, 96 articles from Web of Science, 66 articles from EMBASE, and 99 articles from MEDLINE. We hand-searched bibliographies of retrieved papers and 2 article was included. After removing 248 duplicate documents, 82 papers were deemed ineligible after title and abstract screening, and 22 papers were excluded after further screening through full-text reading. After the exclusion of all the 352 unqualified studies, a total of 14 retrospective case analyses were included in this meta-analysis ${ }^{(10-23)}$. The process of study selection is illustrated in Appendix S1.

\subsection{Study characteristics}

We extracted the features of the literature above. The study included 236 pregnant women with laboratoryconfirmed COVID -19 from December 8, 2019 to April 4, 2020 of whom 160 were in China and 76 were in America. The characteristics of the included literature are presented in Table 1.

\subsection{Assessment of quality}

We evaluated the quality of the fourteen included documents according to IHE case series methodological quality evaluation tool. The result was that the thirteen articles ranged from $45 \%$ to $65 \%$ respectively, all of which were lower than $70 \%$ and were of low quality. Only one article met $70 \%$, considered acceptable quality. These articles were all retrospective studies with few cases and without control group, intervention and blind method, so they were rated as low quality. However, there were only these documents at present, we had no choice but to include them. The literature quality assessment is shown in Table 2 .

\subsection{Quantitative data synthesis}

Because of the high heterogeneity of this study, we used random effects model. Meta-analysis results showed: the incidence of severe case or death was $12 \%, 95 \%$ CI: $0.03^{\sim} 0.20, I^{2}=0 \%, P=0.006$; the incidence of fever was $51 \%, 95 \% \mathrm{CI}: 0.35^{\sim} 0.67, I^{2}=89 \%, P$ ¡0.00001; the incidence of cough was $31 \%, 95 \% \mathrm{CI}: 0.23 \sim 0.39, I$ ${ }^{2}=38 \%, P\left\lceil 0.00001\right.$; the incidence of lymphopenia was $49 \%, 95 \%$ CI: $0.29^{\sim} 0.70, I^{2}=83 \%, P\lceil 0.00001$; the incidence of positive CT findings was $71 \%, 95 \% \mathrm{CI}: 0.49^{\sim} 0.93, I^{2}=90 \%, P ; 0.00001$; the incidence of coexisting disorders was $33 \%, 95 \% \mathrm{CI}: 0.21^{\sim} 0.44, I^{2}=70 \%, P$ ¡ 0.00001 ; the incidence of preterm labor was $23 \%, 95 \% \mathrm{CI}$ : $0.14^{\sim} 0.32, I^{2}=21 \%, P$ i 0.00001 ; the incidence of cesarean section was $65 \%, 95 \%$ CI: $0.42^{\sim} 0.87, I^{2}=90 \%, P$ $¡ 0.00001$; the incidence of fetal distress was $29 \%, 95 \% \mathrm{CI}: 0.08 \sim 0.49, I^{2}=68 \%, P=0.007$; the incidence of neonatal asphyxia or neonatal death or stillbirth was $9 \%, 95 \% \mathrm{CI}$ : $-0.03^{\sim} 0.21, I^{2}=0 \%, P=0.14$; the incidence of neonatal infection was $12 \%, 95 \%$ CI: $-0.01^{\sim} 0.26, I^{2}=0 \%, P=0.06$; SARS-CoV-2 test of breast milk was only mentioned in Chen H 2020.2.12, and the incidence was 0, which cannot be calculated by meta-analysis.

To sum up, the $P$ values of neonatal asphyxia or neonatal death or stillbirth and neonatal infection were both greater than 0.05 , which have no statistical significance. We couldn't calculate the incidence of positive SARS-CoV-2 test in breast milk as well. Otherwise the $P$ values in remaining indicators were all less than 0.05, which was statistically significant. The most common clinical features were positive CT findings (71\%), cesarean section (65\%), and fever (51\%), followed by lymphopenia (49\%), cough (31\%) and severe case or death (12\%). Adverse pregnancy outcomes included coexisting disorders (33\%), fetal distress (29\%) and preterm labor $(23 \%)$, which was in descending order. Among them, the $I^{2}$ value of severe case or death was $0 \%$, which was low heterogeneity. Despite the indicator mentioned above was referred to 10 literatures respectively, the incidences of eight documents were all 0 and there were only two none-zero data of the 
indicator. The $I^{2}$ value of preterm labor was $21 \%$, which was low heterogeneity. The $I^{2}$ value of cough was $38 \%$, which was medium heterogeneity, and the remaining $I^{2}$ value of indicators ranged from $68 \%$ to $90 \%$, which were all high heterogeneity.

Furthermore, we carried out the subgroup analyze based on the data from the fourteen retrospective analyses of COVID-19 infection in pregnant women above and one meta-analysis of epidemiology in all patients infected COVID-19 ${ }^{(24)}$. All patients were divided into two subgroups, namely pregnant women and nonpregnant patients. In the fifteen articles, only two indexes, fever and cough, were coincident, which were analyzed in subgroup. The results were as follows. The incidence of fever in pregnant women group was $51 \%$, which were significant lower than $91 \%$ in nonpregnant patients group $(P ; 0.00001)$. The incidence of cough in pregnant women group was also significant lower than nonpregnant patients $(31 \%$ vs $67 \%, P ; 0.0001)$. The forest plot of subgroup analysis is illustrated in Fig. 1 and Fig. 2.

\subsection{Risk of publication bias}

The funnel plots of fever, cough, positive CT findings and coexisting disorders are symmetric, which meant that these indicators may not be published biased. On the contrary, the funnel plots of severe case or death, lymphopenia, preterm labor, cesarean section, fetal distress, neonatal asphyxia or neonatal death or stillbirth and neonatal infection are asymmetric, which meant that the indicators above may have publication bias. Since there was only 1 article about SARS-CoV-2 test of breast milk, it was meaningless to draw a funnel plot. Therefore, the publication bias of it was not evaluated. The funnel plots of fever and cough are shown in Figure S1 and Figure S2.

\section{Discussion}

\subsection{Main Findings}

The cases discussed in this article were 160 pregnant women with COVID-19 in China and 76 in America. The pooled results of this meta-analysis showed that among the pregnant women with COVID-19, $71 \%$ had positive CT findings, $65 \%$ had cesarean section, $51 \%$ had fever, $49 \%$ had lymphopenia in laboratory examination, $33 \%$ had coexisting disorders, $31 \%$ had cough, $29 \%$ had fetal distress, $23 \%$ had preterm labor and $12 \%$ were severe case or death. Pregnant women with COVID-19 had significantly lower rates of fever and cough than nonpregnant patients with COVID-19.

\subsection{Strengths and Limitations}

Currently there are only meta-analyses of the epidemiology of common patients infected with COVID-19, and there is few meta- analysis to explore the clinical features and outcomes of pregnant women with COVID-19. It is helpful to formulate clinical treatment strategies for pregnant women with COVID-19.

The disadvantage of this article is the small sample and the general quality of included documents, which lowers the credibility of the meta-analysis results. Besides all the included articles are retrospectively case analysis without control group, it also biased the results. Furthermore, the funnel plots showed that most indicators may have publication bias. Most of the included cases are Chinese, and the others are American. There are few reports describing the cases outside the two regions. Further research on pregnant women with COVID-19 worldwide is needed.

\subsection{Interpretation}

The subgroup analysis between pregnant women with COVID-19 and nonpregnant people with COVID-19 showed that the incidences of fever and cough in pregnant women with COVID-19 (51\%, 31\%) were lower than that in ordinary people $(91 \%, 67 \%)$, which may due to the changes in the immune system of pregnant women and further research is needed. A study from China CDC showed, $80.9 \%$ of Chinese patients were considered asymptomatic or mild pneumonia ${ }^{(25)}$.Desmond Sutton et al. showed that of the 215 pregnant women who gave birth at the New York-Presbyterian Allen Hospital and Columbia University Irving Medical Center, $29(87.9 \%)$ of the 33 patients who were positive for Sars-CoV-2 test had no symptoms of COVID-19 at the time of admission ${ }^{(19)}$. The fact that the asymptomatic rate in the infected general population in China 
is lower than in infected pregnant women in the New York Medical Center seems to support this conclusion above. It suggested that Sars-CoV-2 test should be universal in high-risk areas to improve the isolation of asymptomatic infected individuals. It is different from the fact that pregnant women with SARS-CoV have worse prognosis than ordinary people with SARS- $\mathrm{CoV}^{(26)}$. It is possible that in pregnant women, the clinical outcome of COVID-19 infection is better than that of SARS-CoV. Yan et al. confirmed that the current mortality rate of COVID-19(2\%) is significantly lower than that of SARS (9.6\%), which may indicate that SARS is more pathogenic and lethal than COVID-19, thus pregnant women with COVID-19 infection had better outcomes than those with SARS-CoV ${ }^{(27)}$. However, our finding that pregnancy women with COVID-19 had better clinical outcomes, might be biased owing to the relatively small sample included in this meta-analysis.

A meta-analysis showed that the CT positive rate of covid-19 infection in the general population was $89.76 \%{ }^{(28)}$, which was more than $71 \%$ in this paper. This also corresponded to the conclusion above that the clinical outcomes of pregnant women with COVID-19 were superior to general population. The incidence of positive CT findings was the highest among the symptoms. Shital J. Patel et al. confirmed that chest CT was considered a low-dose examination provided the fetus was excluded from the primary beam, and the estimated radiation doses were too low to induce fetal neurologic deficits during any trimester of pregnancy ${ }^{(29)}$. It seemed that chest $\mathrm{CT}$ was suitable for routine screening of patients. However, there were a large quantity of pregnant women with asymptomatic infection $(87.9 \%)^{(19)}$. If chest $\mathrm{CT}$ is used as routine screening, it means that almost all pregnant women need it. In addition, WHO defines screening as the presumptive identification of unrecognized disease in an apparently healthy, asymptomatic population by means of tests, examinations or other procedures that can be applied rapidly and easily to the target population ${ }^{(30)}$. As a consequence, it is improper to perform chest CT as a screening tool for pregnant women with COVID-19. We recommend to use it as the routine examination for suspected cases.

The rate of preterm labor in normal pregnant women worldwide is about $11 \%{ }^{(31)}$, which is lower than the result of this article $(23 \%)$. The possible reason is that women in the third trimester of pregnancy terminate their pregnancy early after being infected with COVID-19 in order to proceed with further treatment. Most of these women choose early delivery by cesarean section in order to avoid prolonged labor which may worse COVID-19 for pregnant women ${ }^{(32)}$ and increased risk of infection for medical staff( ${ }^{(33)}$. Chen $\mathrm{R}$ et al. confirmed that both epidural anesthesia and general anesthesia were safe and effective for women with COVID-19 during cesarean section ${ }^{(12)}$.

Due to the $P$ value was greater than 0.05 , the rate of neonatal COVID-19 infection should not be considered. Wang $\mathrm{S}$ et al. reported the first case in China that the mother with COVID-19 gave birth to an infected baby in February 2, $2020{ }^{(34)}$ and the instant SARS-CoV-2 nucleic acid tests of umbilical cord blood and placenta were both negative. And there are 3 neonates infected in the included literature. Khan S. et al reported that the swab samples tested within 24 hours after the delivery were positive in two neonates while intrauterine tissue samples such as placenta, cord blood or amniotic fluid were not tested ${ }^{(14)}$. Yu $\mathrm{N}$ et al reported that the nucleic acid test for the throat swab of one neonate was positive at $36 \mathrm{~h}$ after birth ${ }^{(22)}$. Without testing the intrauterine tissue samples, we could not confirm if the COVID-19 infection in the neonate was the result of intrauterine transmission. Two studies also showed that the test for SARS-CoV-2 specific antibodies (IgG and IgM) in neonatal serum samples could be evidence of vertical transmission ${ }^{(35,36)}$. Other literature revealed that almost all the other newborns from infected women were tested negative for SARS$\mathrm{CoV}-2^{(10-13,15-21,23,37-40)}$. Wang $\mathrm{C}$ et al summarized that there was currently no evidence for intrauterine infection caused by vertical transmission in women with COVID-19 during the third trimester of pregnancy but it was uncertain whether there could be a risk of vertical transmission when the COVID-19 infection occurs in the first or second trimester, or when there was a long clinical manifestation-to-delivery interval (41). So we must continue to keep alert.

\section{Conclusion}

The incidence of fever, cough and positive CT findings in pregnant women with COVID-19 is less than that in the general population. And the rate of preterm labor in normal pregnant women worldwide is lower than 
that in pregnant women with COVID-19. There is no evidence that COVID-19 can propagate vertically for the time being. The conclusion above is possibly helpful to formulate clinical treatment strategies for pregnant women infected with COVID-19.

\section{Author contributions}

LY and JS.Z conducted the literature search, selected studies. YX.Y assessed the methodologic quality of studies. ML and HB.Y extracted data. YJ.G conceived and planned the review, assessed the methodologic quality of studies, verified the data, and drafted and revised the manuscript. RZ provided methodologic advice, content expertise, and revised the manuscript. All authors contributed to writing of the protocol.

\section{Funding}

This work was supported by the National Natural Science Foundation of China (No. 81571465, No. 81871175). The mentioned foundation had no rule in the study design, date analysis, drafting the manuscript, or decision to submit this article for publication.

\section{Conflicts of interest}

The authors have no conflicts of interest.

\section{References}

1. World Health Organization. Novel coronavirus - China. [Internet]. Jan 12, 2020 [cited April 16, 2020]. Available from:https://www.who.int/csr/don/12-january-2020-novel-coronavirus-china/en/.

2. Hui DS, E IA, Madani TA, Ntoumi F, Kock R, Dar O, et al. The continuing 2019-nCoV epidemic threat of novel coronaviruses to global health - The latest 2019 novel coronavirus outbreak in Wuhan, China. International journal of infectious diseases. 2020;91:264-6.

3. World Health Organization. WHO Director-General's remarks at the media briefing on 2019-nCoV on 11 February 2020. [Internet]. Feb 11, 2020 [cited April 16, 2020]. Available from:https://www.who.int/dg/speeches/detail/whodirector-general-s-remarks-at-the-media-briefing-on-2019-ncov-on-11-february-2020.

4. DXY.DX Doctor. COVID-19 Global Pandemic Real-time Report. [Internet]. [cited April 16, 2020]. Available from:https://ncov.dxy.cn/ncovh5/view/pneumonia?from $=d x y \&$ ssource $=$ Élink=ESshare $=$.

5. World Health Organization. Summary of probable SARS cases with onset of illness from 1 November 2002 to 31 July 2003. [Internet]. Dec 31, 2003 [cited April 16, 2020]. Available from:https://www.who.int/csr/sars/country/table20 04-21/en/.

6. Stroup DF, Berlin JA, Morton SC, Olkin I, Williamson GD, Rennie D, et al. Meta-analysis of observational studies in epidemiology: a proposal for reporting. Meta-analysis Of Observational Studies in Epidemiology (MOOSE) group. Jama. 2000;283(15):2008-12.

7. Moher D, Shamseer L, Clarke M, Ghersi D, Liberati A, Petticrew M, et al. Preferred reporting items for systematic review and meta-analysis protocols (PRISMA-P) 2015 statement. Systematic reviews. $2015 ; 4(1): 1$.

8. Wang XQ, Chen YL, Qu QY, Yao L, Wei D, Wang Q, et al. Interpretation of methodological quality assessment tools for case series studies. Chinese Journal of Evidence-Based Pediatrics. 2015;5(10). doi: 10.3969/j.issn.1673-5501.2015.05.012.

9. Chen YH, Du L, Geng XY, Liu GJ. Implement Meta-Analysis with Non-Comparative Binary Data in RevMan Soft ware. Chinese Journal of Evidence-based Medicine. 2014;14(7):889-96.

10. Breslin N, Baptiste C, Gyamfi-Bannerman C, Miller R, Martinez R, Bernstein K, et al. COVID-19 infection among asymptomatic and symptomatic pregnant women: Two weeks of confirmed presentations to an affiliated pair of New York City hospitals. American journal of obstetrics \& gynecology MFM. 2020:100118. [Epub ahead of print]. doi: 10.1016/j.ajogmf.2020.100118. 
11. Chen H, Guo J, Wang C, Luo F, Yu X, Zhang W, et al. Clinical characteristics and intrauterine vertical transmission potential of COVID-19 infection in nine pregnant women: a retrospective review of medical records. Lancet. 2020;395(10226):809-15.

12. Chen R, Zhang Y, Huang L, Cheng BH, Xia ZY, Meng QT. Safety and efficacy of different anesthetic regimens for parturients with COVID-19 undergoing Cesarean delivery: a case series of 17 patients. Canadian journal of anaesthesia. 2020;1-9.

13. Chen S, Liao E, Cao D, Gao Y, Sun G, Shao Y. Clinical analysis of pregnant women with 2019 novel coronavirus pneumonia. Journal of medical virology. 2020. [Epub ahead of print]. doi: 10.1002/jmv.25789.

14. Khan S, Jun L, Nawsherwan, Siddique R, Li Y, Han G, et al. Association of COVID-19 infection with pregnancy outcomes in healthcare workers and general women. Clinical microbiology and infection. 2020;S1198-743X(20)30180-4. [Epub ahead of print]. doi: 10.1016/j.cmi.2020.03.034.

15. Li N, Han L, Peng M, Lv Y, Ouyang Y, Liu K, et al. Maternal and neonatal outcomes of pregnant women with COVID-19 pneumonia: a case-control study. Clinical infectious diseases. 2020. [Epub ahead of print]. doi: $10.1093 / \mathrm{cid} / \mathrm{ciaa352}$.

16. Liu D, Li L, Wu X, Zheng D, Wang J, Yang L, et al. Pregnancy and Perinatal Outcomes of Women With Coronavirus Disease (COVID-19) Pneumonia: A Preliminary Analysis. American journal of roentgenology. 2020:1-6.

17. Liu H, Liu F, Li J, Zhang T, Wang D, Lan W. Clinical and CT Imaging Features of the COVID-19 Pneumonia: Focus on Pregnant Women and Children. The Journal of infection. 2020;80(5):e7-e13.

18. Liu Y, Chen H, Tang K, Guo Y. Clinical manifestations and outcome of SARS-CoV-2 infection during pregnancy. The Journal of infection. 2020. [Epub ahead of print]. doi: 10.1016/j.jinf.2020.02.028.

19. Sutton D, Fuchs K, D'Alton M, Goffman D. Universal Screening for SARS-CoV-2 in Women Admitted for Delivery. The New England journal of medicine. 2020. [Epub ahead of print]. doi: 10.1056/NEJMc2009316.

20. Wu X, Sun R, Chen J, Xie Y, Zhang S, Wang X. Radiological findings and clinical characteristics of pregnant women with COVID-19 pneumonia. International journal of gynaecology and obstetrics. 2020. [Epub ahead of print]. doi: 10.1002/ijgo.13165.

21. Yang H, Sun G, Tang F, Peng M, Gao Y, Peng J, et al. Clinical Features and Outcomes of Pregnant Women Suspected of Coronavirus Disease 2019. The Journal of infection. 2020;S0163-4453(20)30212-7. [Epub ahead of print]. doi: 10.1016/j.jinf.2020.04.003.

22. Yu N, Li W, Kang Q, Xiong Z, Wang S, Lin X, et al. Clinical features and obstetric and neonatal outcomes of pregnant patients with COVID-19 in Wuhan, China: a retrospective, single-centre, descriptive study. The Lancet Infectious diseases. 2020;S1473-3099(20)30176-6. [Epub ahead of print]. doi: 10.1016/S14733099(20)30176-6.

23. Zhu H, Wang L, Fang C, Peng S, Zhang L, Chang G, et al. Clinical analysis of 10 neonates born to mothers with 2019-nCoV pneumonia. Translational pediatrics. 2020;9(1):51-60.

24. Yang J, Zheng Y, Gou X, Pu K, Chen Z, Guo Q, et al. Prevalence of comorbidities in the novel Wuhan coronavirus (COVID-19) infection: a systematic review and meta-analysis. International journal of infectious diseases. 2020;S1201-9712(20)30136-3. [Epub ahead of print]. doi: 10.1016/j.ijid.2020.03.017.

25. Novel Coronavirus Pneumonia Emergency Response Epidemiology Team. The epidemiological characteristics of an outbreak of 2019 novel coronavirus diseases (COVID-19) in China. Zhonghua liu xing bing xue za zhi. 2020;41(2):145-51.

26. Lam CM, Wong SF, Leung TN, Chow KM, Yu WC, Wong TY, et al. A case-controlled study comparing clinical course and outcomes of pregnant and non-pregnant women with severe acute respiratory syndrome. BJOG. 2004;111(8):771-4. 
27. Yan Y, Shin WI, Pang YX, Meng Y, Lai J, You C, et al. The First 75 Days of Novel Coronavirus (SARSCoV-2) Outbreak: Recent Advances, Prevention, and Treatment. International journal of environmental research and public health. 2020;17(7).

28. Bao C, Liu X, Zhang H, Li Y, Liu J. Coronavirus Disease 2019 (COVID-19) CT Findings: A Systematic Review and Meta-analysis. Journal of the American College of Radiology. 2020;S1546-1440(20)30262-3. [Epub ahead of print]. doi: 10.1016/j.jacr.2020.03.006.

29. Patel SJ, Reede DL, Katz DS, Subramaniam R, Amorosa JK. Imaging the pregnant patient for nonobstetric conditions: algorithms and radiation dose considerations. Radiographics. 2007;27(6):1705-22.

30. World Health Organization. Cancer: Screening. [Internet]. [cited April 16, 2020].Available from:https://www.who.int/can screening/screening/en/.

31. Vogel JP, Chawanpaiboon S, Moller AB, Watananirun K, Bonet M, Lumbiganon P. The global epidemiology of preterm birth. Best practice \& research. Clinical obstetrics \& gynaecology. 2018;52:3-12.

32. Chen D, Yang H, Cao Y, Cheng W, Duan T, Fan C, et al. Expert consensus for managing pregnant women and neonates born to mothers with suspected or confirmed novel coronavirus (COVID-19) infection. International journal of gynaecology and obstetrics. 2020;149(2):130-136.

33. Bauer M, Bernstein K, Dinges E, Delgado C, El-Sharawi N, Sultan P, et al. Obstetric Anesthesia During the COVID-19 Pandemic. Anesthesia and analgesia. 2020. [Epub ahead of print]. doi: 10.1213/ANE.0000000000004856.

34. Wang S, Guo L, Chen L, Liu W, Cao Y, Zhang J, et al. A case report of neonatal COVID-19 infection in China. Clinical infectious diseases. 2020;ciaa225. [Epub ahead of print]. doi: 10.1093/cid/ciaa225.

35. Dong L, Tian J, He S, Zhu C, Wang J, Liu C, et al. Possible Vertical Transmission of SARSCoV-2 From an Infected Mother to Her Newborn. Jama. 2020;e204621. [Epub ahead of print]. doi: 10.1001/jama.2020.4621.

36. Zeng H, Xu C, Fan J, Tang Y, Deng Q, Zhang W, et al. Antibodies in Infants Born to Mothers With COVID-19 Pneumonia. Jama. 2020;e204861. [Epub ahead of print]. doi: 10.1001/jama.2020.4861.

37. Chen S, Huang B, Luo DJ, Li X, Yang F, Zhao Y, et al. Pregnant women with new coronavirus infection: a clinical characteristics and placental pathological analysis of three cases. Chinese journal of pathology. 2020;49(0):E005.

38. Fan C, Lei D, Fang C, Li C, Wang M, Liu Y, et al. Perinatal Transmission of COVID-19 Associated SARS-CoV-2: Should We Worry? Clinical infectious diseases. 2020;ciaa226. [Epub ahead of print]. doi: $10.1093 / \mathrm{cid} / \mathrm{ciaa226}$.

39. Wang X, Zhou Z, Zhang J, Zhu F, Tang Y, Shen X. A case of 2019 Novel Coronavirus in a pregnant woman with preterm delivery. Clinical infectious diseases. 2020;ciaa200. [Epub ahead of print]. doi: $10.1093 / \mathrm{cid} /$ ciaa200.

40. Wen R, Sun Y, Xing QS. A patient with SARS-CoV-2 infection during pregnancy in Qingdao, China. Journal of microbiology, immunology, and infection. 2020. [Epub ahead of print]. doi: 10.1016/j.jmii.2020.03.004.

41. Wang C, Zhou YH, Yang HX, Poon LC. Intrauterine vertical transmission of SARS-CoV-2: what we know so far. Ultrasound in obstetrics \& gynecology. 2020. [Epub ahead of print]. doi: 10.1002/uog.22045.

Table 1 Characteristics of the studies included in the meta-analysis 


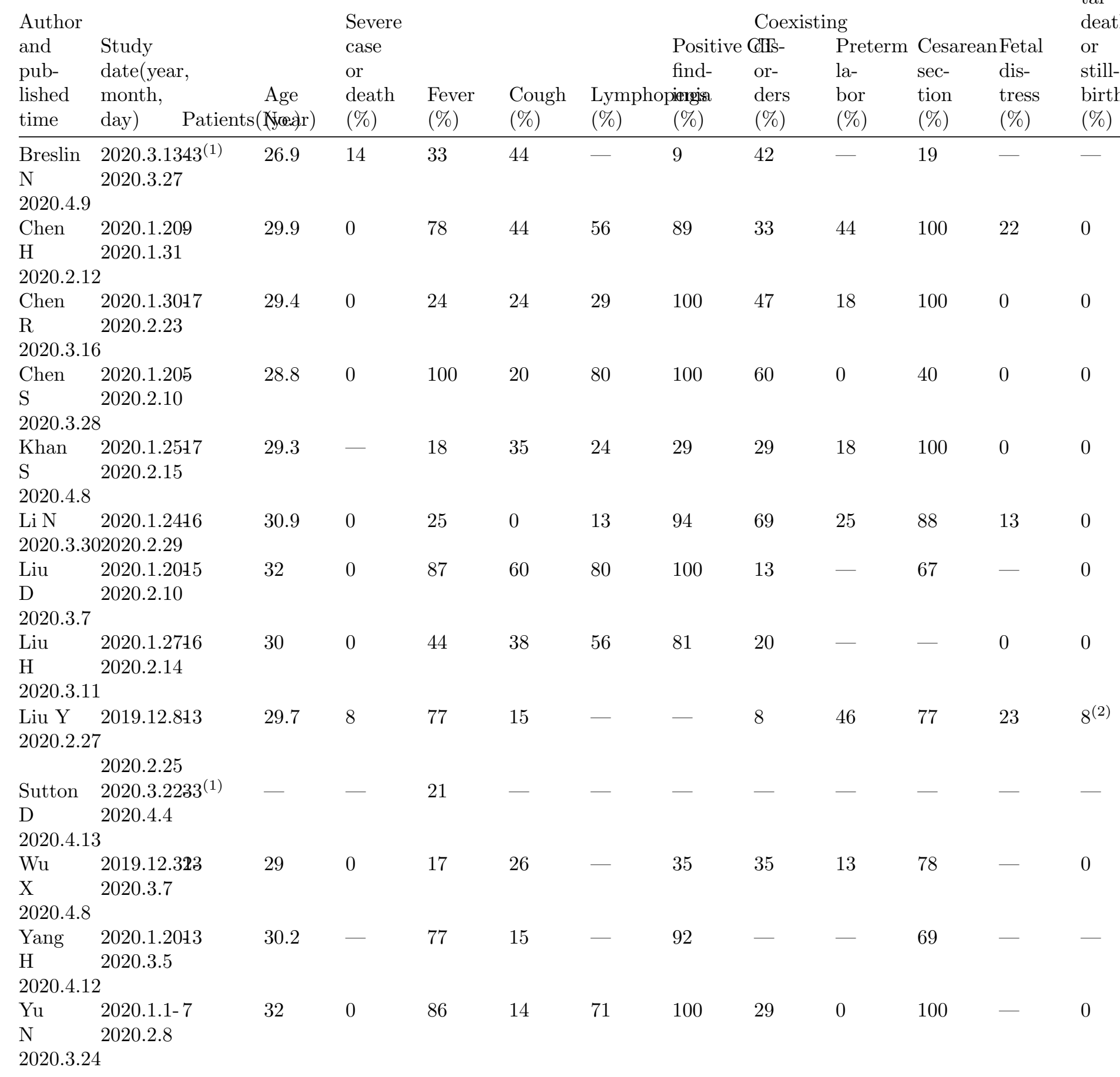




\begin{tabular}{|c|c|c|c|c|c|c|c|c|c|c|c|}
\hline \multirow{2}{*}{$\begin{array}{l}\text { Author } \\
\text { and } \\
\text { pub- } \\
\text { lished } \\
\text { time }\end{array}$} & \multirow[b]{2}{*}{$\begin{array}{l}\text { Study } \\
\text { date(year, } \\
\text { month, } \\
\text { day) I }\end{array}$} & \multirow[b]{2}{*}{$\begin{array}{c}\text { Age } \\
\text { Patients(Nya)r) }\end{array}$} & \multirow{2}{*}{$\begin{array}{l}\text { Severe } \\
\text { case } \\
\text { or } \\
\text { death } \\
(\%)\end{array}$} & \multirow[b]{2}{*}{$\begin{array}{l}\text { Fever } \\
(\%)\end{array}$} & \multirow[b]{2}{*}{$\begin{array}{l}\text { Cough } \\
(\%)\end{array}$} & \multicolumn{6}{|c|}{ Coexisting } \\
\hline & & & & & & $\begin{array}{l}\text { Lymphopi } \\
(\%)\end{array}$ & $\begin{array}{l}\text { Positiv } \\
\text { find- } \\
\text { piergis } \\
(\%)\end{array}$ & $\begin{array}{l}\text { Clis- } \\
\text { or- } \\
\text { ders } \\
(\%)\end{array}$ & $\begin{array}{l}\text { Preterm } \\
\text { la- } \\
\text { bor } \\
(\%)\end{array}$ & $\begin{array}{l}\text { Cesarean } \\
\text { sec- } \\
\text { tion } \\
(\%)\end{array}$ & $\begin{array}{l}\text { Fetal } \\
\text { dis- } \\
\text { tress } \\
(\%)\end{array}$ \\
\hline $\begin{array}{l}\text { Zhu } \\
\text { H } \\
2020.2\end{array}$ & $\begin{array}{l}2020.1 .209 \\
2020.2 .5\end{array}$ & 30 & - & 89 & 44 & - & 100 & 0 & 33 & 78 & 16 \\
\hline
\end{tabular}

(1) The patients are from American hospitals in the two literatures, and others are from Chinese hospitals.

(2) 1 case was stillbirth.

(3) 1 case was neonatal death.

Table 2 Literature quality assessment with IHE case series methodological quality evaluation tool

\begin{tabular}{lllll}
\hline & Research purpose & Research population & Intervention and joint intervention & Outcome measures \\
\hline Breslin N 2020.4.9 & 1 & 5 & 0 & 2 \\
Chen H 2020.2.12 & 1 & 4 & 0 & 2 \\
Chen R 2020.3.16 & 1 & 4 & 1 & 3 \\
Chen S 2020.3.28 & 1 & 4 & 0 & 2 \\
Khan S 2020.4.8 & 1 & 4 & 0 & 2 \\
Li N 2020.3.30 & 1 & 4 & 0 & 2 \\
Liu D 2020.3.7 & 1 & 4 & 0 & 2 \\
Liu H 2020.3.11 & 1 & 4 & 0 & 2 \\
Liu Y 2020.2.27 & 1 & 5 & 0 & 2 \\
Sutton D 2020.4.13 & 1 & 3 & 0 & 2 \\
Wu X 2020.4.8 & 1 & 4 & 0 & 2 \\
Yang H 2020.4.12 & 1 & 4 & 0 & 2 \\
Yu N 2020.3.24 & 1 & 4 & 0 & 2 \\
Zhu H 2020.2 & 1 & 5 & 0 & 2 \\
\hline
\end{tabular}

\section{Hosted file}

Figure 1, 2.docx available at https://authorea.com/users/313307/articles/443841-clinical-featuresand-outcomes-of-pregnant-women-with-covid-19-a-systematic-review-and-meta-analysis 